\title{
Technique to stabilize Wax Occlusion Rims following Maxillomandibular Relationship Records
}

\author{
${ }^{1}$ Pravinkumar Patil, ${ }^{2}$ Smita Nimbalkar, ${ }^{3}$ Sameera Shaikh
}

\begin{abstract}
Maxillomandibular relationship (MMR) recorded with the help of wax occlusion rims, with or without an interocclusal recording medium, tends to distort during the mounting procedure under higher room temperature. When the records were required to be transported to the distant laboratories for mounting, maintaining the sealed occlusion rims undistorted is always a concern to the prosthodontist. A simplified technique for stabilizing the MMR records made with wax occlusion rims is described. This technique is easy to perform and no special instruments or materials are required. The records stabilized with this technique remain undistorted till mounting even under high room temperature and can safely be transported to the distant laboratories for mounting.
\end{abstract}

Keywords: Complete denture, Maxillomandibular relationship record, Occlusion rims.

How to cite this article: Patil P, Nimbalkar S, Shaikh S. Technique to stabilize Wax Occlusion Rims following Maxillomandibular Relationship Records. Int J Prosthodont Restor Dent 2017;7(4):114-116

Source of support: Nil

Conflict of interest: None

\section{INTRODUCTION}

Accuracy of MMR record is of utmost importance in the construction of complete dentures. ${ }^{1}$ Several methods have been described to record MMR for fabrication of complete dentures. ${ }^{2-4}$ Of these techniques, the direct interocclusal record using wax occlusion rims on record bases remains the most popular technique, even though it still needs considerable clinical time to adjust the height of the occlusion rims to match the desired vertical height of the face. ${ }^{5}$ In association with wax occlusion rims, several interocclusal

\footnotetext{
${ }^{1,2}$ Senior Lecturer, ${ }^{3}$ Private Practitioner

${ }^{1}$ Division of Clinical Dentistry, School of Dentistry, International Medical University, Kuala Lumpur, Malaysia

${ }^{2}$ Department of Orthodontics, Faculty of Dentistry, MAHSA University, Bandar Saujana Putra, Jenjarum, Selangor, Malaysia

${ }^{3}$ Department of Prosthodontics, Private Practice, Mumbai Maharashtra, India

Corresponding Author: Pravinkumar Patil, Senior Lecturer Division of Clinical Dentistry, School of Dentistry, International Medical University, Kuala Lumpur, Malaysia, Phone: +601135022042, e-mail: pravinandsmita@yahoo.co.in
}

recording materials have also been used to record MMR in edentulous patients. ${ }^{6,7}$ The MMR recorded with the help of the wax occlusion rims (with or without interocclusal recording medium) tends to distort during mounting procedure under higher room temperature, especially in tropical countries where the temperature usually remains between 40 and $48^{\circ} \mathrm{C}$ in summer. When the MMR records required to be transported to the distant laboratories for mounting, maintaining the sealed occlusion rims (MMR records) undistorted is always a concern to the prosthodontist. A simplified technique for stabilizing the MMR records made with wax occlusion rims is described.

\section{PROCEDURE}

- Fabricate maxillary and mandibular record bases with autopolymerizing acrylic resin on the respective final casts using the wax template technique described by Zarb and Finer. ${ }^{8}$

- Take the direct MMR record using the occlusion rims prepared with the baseplate wax on record bases in usual manner (Fig. 1).

- Orient the maxillary cast on the semi-adjustable articulator (Hanau H2; Teledyne Technologies, Los Angeles, CA) using face bow transfer before sealing the MMR record.

- Hold the maxillary and mandibular occlusion rims in original MMR and mark two vertical lines (parallel and $1.5 \mathrm{~cm}$ apart from each other) on buccal surface of the completed wax-MMR record as shown in Figure 2.

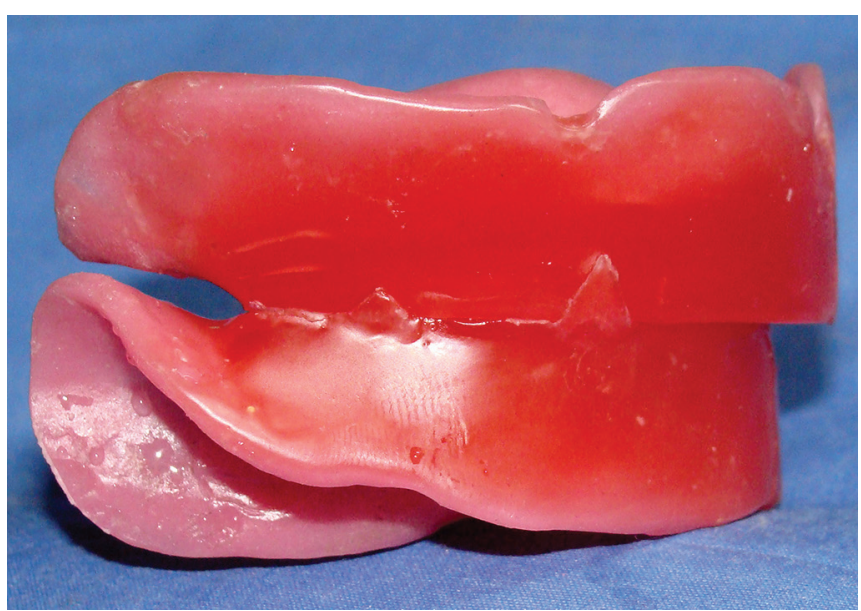

Fig. 1: Completed MMR record made with wax occlusion rims 


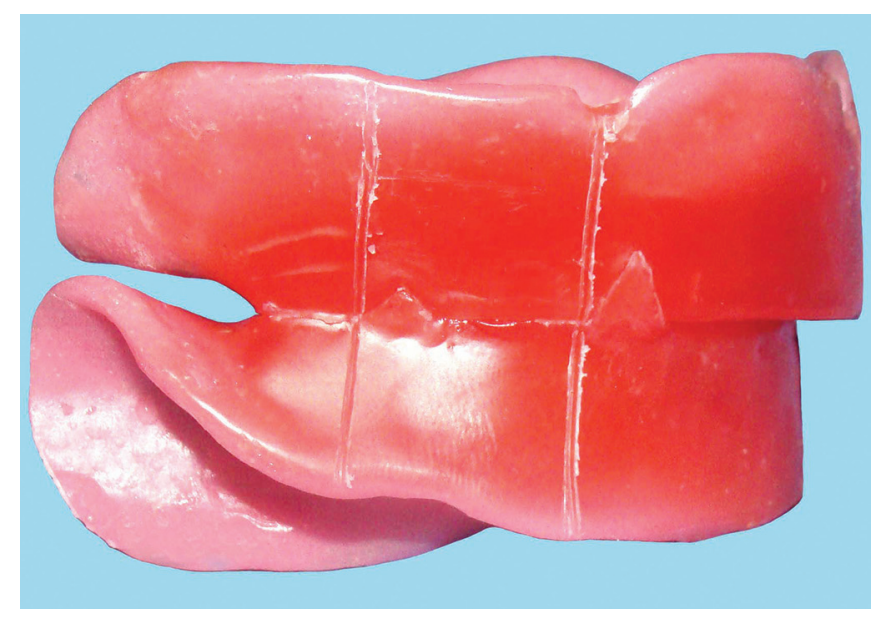

Fig. 2: Two parallel lines scraped indicating area of acrylic resin layer for stabilization

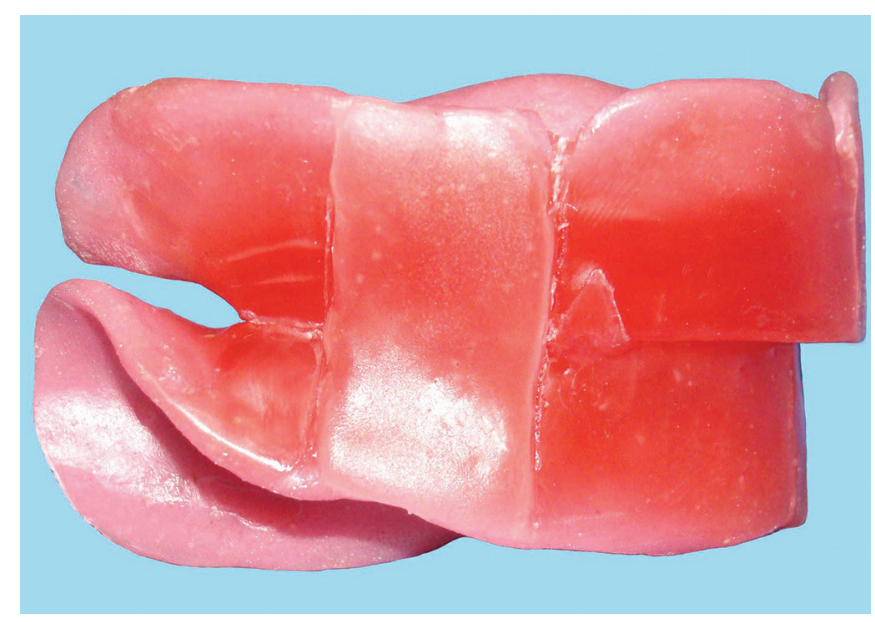

Fig. 4: Completed acrylic resin application

- Remove the wax of $2 \mathrm{~mm}$ in width parallel to the borders (between the two marked lines and 2-3 mm away from the borders) to expose underlying resin surfaces of the maxillary and mandibular record bases (Fig. 3). Make sure that exposed area is completely free of wax. Care must be taken during removal of the wax to prevent distortion of the MMR record.

- Mix and apply clear autopolymerizing acrylic resin on buccal surface of the wax occlusion rims between the marked lines to make a 2-mm-thick layer. Apply the mixed acrylic resin in two to three sections to prevent the distortion of the MMR records due to shrinkage of the polymerization shrinkage of the resin. Make sure that exposed portions of the record bases are completely filled with the clear acrylic resin. Prevent the seepage of mixed acrylic resin over the borders.

- After completion of polymerization of the clear autopolymerizing acrylic resin layer (Fig. 4), repeat steps 4 to 6 to add similar layer on the opposite side of the MMR record.

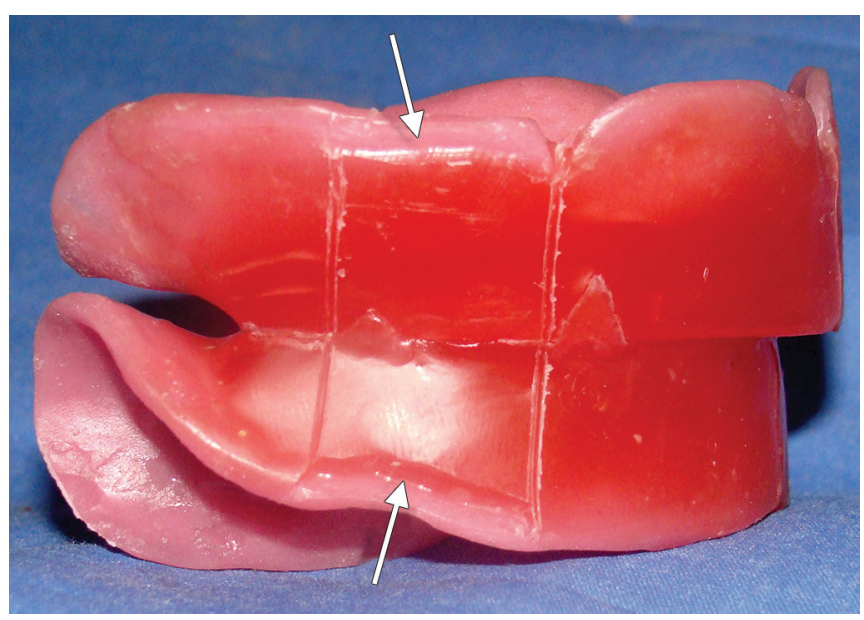

Fig. 3: Arrows indicating border areas freed from wax for acrylic resin application

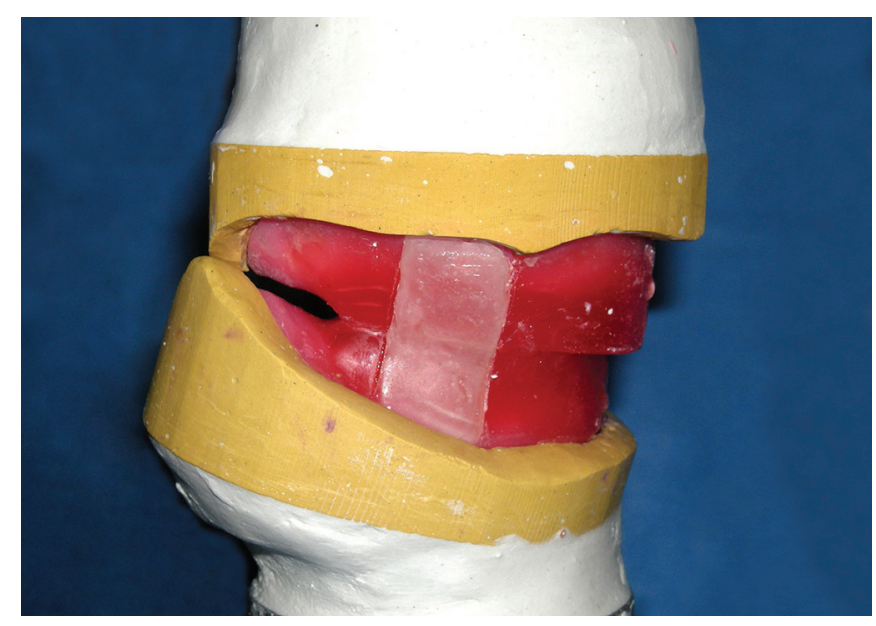

Fig. 5: Completed mounting

- Mount the mandibular cast onto the semi-adjustable articulator using the MMR record in usual manner (Fig. 5). Note that the MMR record sealed with this technique is sufficiently stable and remains undistorted even under high room temperature.

- Remove the clear acrylic resin layer from the MMR record by trimming and separating the layer from the record bases with the help of round carbide bur (EL4; SS White Burs, Lakewood, New Jersey, USA) (Fig. 6). Finish the trimmed portion in usual manner and proceed with the teeth arrangement.

\section{DISCUSSION}

This technique is easy to perform and no special instruments or materials are required. The MMR records stabilized with this technique remain undistorted till mounting even under high room temperature and can safely be transported to the distant laboratories for mounting. The most important problem is of wax distortion. Even in normal temperature, the chances of MMR 


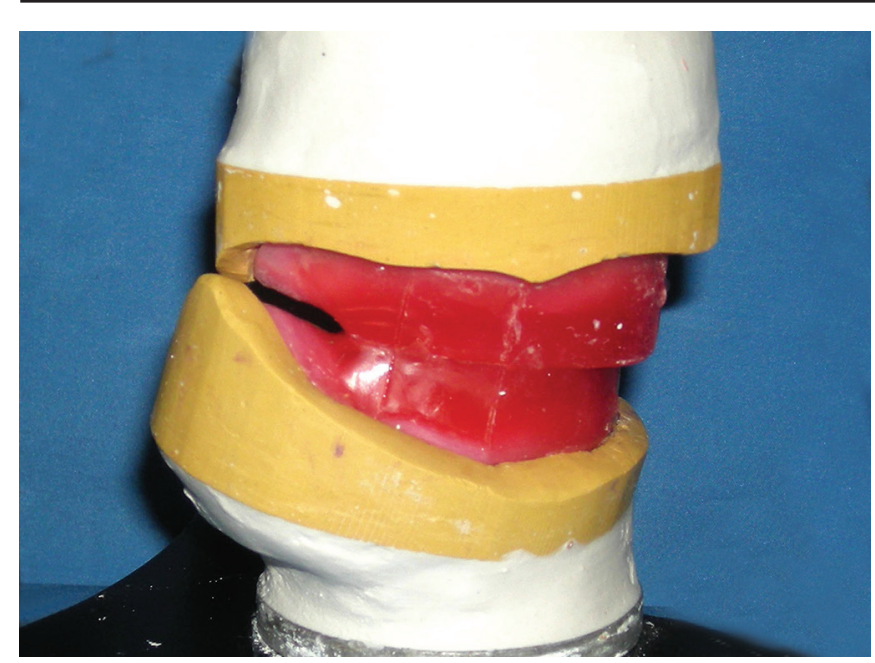

Fig. 6: Acrylic resin layer removed after completion of mounting

distortion are high during transport of the records to the laboratory. The acrylic resin layer applied to stabilize the occlusion rims can be easily removed once the mounting procedure is completed. Hence, the presented technique describes a very simple procedure to avoid unwanted record distortion. The MMR record distortion can only be diagnosed at the try-in appointment. A new MMR record is required in situations where the mounting is done with the distorted MMR. Hence, to avoid repetition of the procedures and to save patient and clinician's time, the described technique is much helpful. There is no disadvantage of this technique except the additional time required for sealing the occlusion rims with acrylic resin. Further research is suggested to evaluate the accuracy of this technique compared with conventional techniques.

\section{REFERENCES}

1. Boulos PJ. Simplified method for recording maxillomandibular relations in complete dentures. N Y State Dent J 2007 Apr;73(3):24-27.

2. Langer A, Michman J. Intraoral technique for recording vertical horizontal maxillomandibular relations in complete dentures. J Prosthet Dent 1969 Jun;21(6):599-606.

3. Ismail $\mathrm{YH}$, George WA. The consistency of the swallowing technique in determining occlusal vertical relation in edentulous patients. J Prosthet Dent 1968 Mar;19(3):230-236.

4. Millet C, Jeannin C, Vincent B, Malquarti G. Report on the determination of occlusal vertical dimension and centric relation using swallowing in edentulous patients. J Oral Rehabil 2003 Nov;30(11):1118-1122.

5. Jaggers JH, Javid NS, Colaizzi FA. Complete denture curriculum survey of dental schools in the United States. J Prosthet Dent 1985 May;53(5):736-739.

6. Urstein M, Fitzig S, Moskona D, Cardash HS. A clinical evaluation of materials used in registering interjaw relationships. J Prosthet Dent 1991 Mar;65(3):372-377.

7. Müller J, Götz G, Hörz W, Kraft E. Study of the accuracy of different recording materials. J Prosthet Dent 1990 Jan;63(1): 41-46.

8. Zarb GA, Finer Y. Identification of shape and location of arch form: The occlusion rim and recording of trial denture base. In: Zarb GA, Bolender CL, Eckert SE, Fenton AH, Jacob RF, Merickske-Stern R, editors. Prosthodontic treatment for edentulous patients: complete dentures and implant supported prostheses. 12th ed. St. Louis: Mosby; 2005. pp. 252-267. 